\title{
Fock-Space methods for Diffusion: Capturing Volume Exclusion via Fermionic Statistics
}

\author{
Gerson C. Duarte-Filho \\ Departamento de Física - Universidade Federal de Sergipe, 49100-000, São Cristóvão, Sergipe, Brazil \\ Fernando A. N. Santos \\ Departamento de Matemática Universidade Federal de Pernambuco, 50670-901, Recife, PE, Brazil and \\ Laboratório de Física Teórica e Computacional, Departamento de Física, \\ Universidade Federal de Pernambuco, 50670-901 Recife, PE, Brazil \\ Eamonn A. Gaffney \\ Wolfson Centre for Mathematical Biology, Mathematical Institute, \\ University of Oxford, Woodstock Road, Oxford, OX2 6GG, UK
}

(Dated: September 28, 2020)

\begin{abstract}
Volume exclusion and single file diffusion play an important role at very small scales, such as those associated with molecular machines, ion channels and transport in zeolites, while introducing fundamental differences compared to Brownian motion, such as changes to the power law relation between the mean square displacement and time. In this work, we map the chemical master equation for excluded diffusion onto a Schrödinger equation via annihilation and creation ladder operators with Fermionic statistics, together with linear and symbolic algebra with the resulting Fock space representation to describe the effect of volume exclusion processes in finite one-dimensional chains. We contrast the dynamics with the non-exclusive (Bosonic) diffusion for crowded, intermediate and dilute particle populations. Motivated by shuttling in molecular machines we proceed to investigate differences in exit time distributions introduced by volume exclusion, incorporating the presence of transport bias. More generally, this study demonstrates how one can analyze volume excluded transport for small stochastic systems, without the need for stochastic simulation and ensemble averaging, simply by considering anti-commutation relations and Fermionic statistics in a Fock space representation of the stochastic dynamics.
\end{abstract}

Keywords: Diffusion; Fermionic Symbolic Algebra; Volume exclusion, Fock Space; Linear Algebra

\section{INTRODUCTION}

Random walks have been investigated since at the least the mid-17th century, with Pascal's study of gambler's ruin [1], though the concept of exclusion for multiple random walkers was first explored by W. Kuhn in the 1930s for volume excluded polymer theory and subsequently developed in-depth by P. J. Flory [2]. More recently, exclusion for random walks, and the associated diffusion processes, has received extensive attention in life-science modelling since biological agents are generally large or moving in confined regions, with reported examples from cell population dynamics and transport in the cell nucleus $[3,4]$. Further recent examples of exclusion effects arise in the phenomenon of single file diffusion processes [5], which constitute one dimensional random walks where walkers cannot pass each other; examples of single file diffusion in small systems, which will be of particular interest in this paper, include ion channel dynamics [6, 7], molecular machines and molecular motor transport $[8,9]$.

There is a diverse array of approaches to incorporate exclusion effects within models of transport. These can be broadly classified as off-lattice, whereby there is no spatial discreteness in the location of random walkers, with examples including: Langevin approaches [10-12], Fokker-Planck equations [11, 12] and explicitly taking the continuum limits of discrete models [13, 14]. Such models can also be analyzed numerically with diverse analytical techniques, for example reconstructing propagators by using images [15] and the Bethe Ansatz [16].

In contrast, there are also on-lattice models, with walkers residing at spatial discrete locations, which is often a convenient mathematical idealisation or, for instance, can represent a random walker transversing between binding sites or potential wells. Examples of frameworks used to study such systems include the discrete EdwardWilkinson equations [17], Gillespie simulations and coupled ordinary differential equations derived from the continuous master equation, each of which describes the dynamics in a lattice compartment $[18,19]$.

A common simplification in many of the single-file diffusion studies is to recognise firstly that two excluding random walkers on approaching each other cannot occupy the same location and thus effectively bounce. In addition, this is very similar to two non-excluding random walkers simply passing through each other, assuming all particles are identical, though this requires neglecting the instant of passing where both walkers occupy the same volume. A particularly elegant example of this 
approximation is highlighted in the review by Bressloff and Newby [8], following the work of Barkai and Silbey [20]. In particular, for large time, unbounded domains and fixed particle number that are initially clustered one finds that the statistics of particle occupancy will relax to a state that does not depend on whether there is exclusion [8].

However, for small domains such approximations may not be valid. Furthermore, numerous other aspects of individual or tagged particle dynamics is contingent on the presence of exclusion; for instance on very large domains with uniform initial distributions of excluding walkers, the mean square displacement of a walker scales with $t^{1 / 2}$, where $t$ is time $[8,20]$. This is sub-diffusive and fundamentally different from the linear $t$ scaling of the mean square displacement for random walkers which can pass each other, emphasizing that exclusion can produce fundamentally different statistics for an individual particle, and by extension, correlations of particles too, while similar remarks apply for particle escape times, as illustrated by long time off-lattice studies [21].

In this study we therefore focus on diffusive dynamics in the presence of exclusion within small single file systems, including the short time dynamics and assess the impact of exclusion. In particular, we rely on the mapping between the chemical master equation and quantum systems to consider the stochastics of on-lattice excluded diffusion in terms of Fock space [22, 23]. This map has been applied in a large variety of problems, such as birthdeath processes [24], diffusion-reaction processes [25-27], gene expression problems [28], stochastic models in population biology [29], enzyme kinetics (Santos et. al. [30]), and Lévy flights [31]. The fundamental concept of this method, often referred to as stochastic mechanics to emphasise the similarity with quantum mechanics, is the analogy of the classical master equation with the evolution equation for a quantum system of interacting particles, as recently reviewed by Baez and Biamonte [32].

In particular, the stochastic mechanics methodology provides analytical tractability for very small systems and the prospect of using symbolic or linear algebra more generally to study system dynamics in depth. This avoids the need for simulating ensembles to generate probability distributions, which more generally offers a means of efficient parameter estimation, Bayesian inference studies and model selection in comparing models with observations, especially when rare but important stochastic events can occur.

Here we develop a study of single file diffusion by considering the changes induced by volume exclusion for diffusion on a linear chain, using stochastic mechanics. Following a simple test case we consider an exemplar motivated from experimental studies of Rotaxane molecular walkers [9], moving across a small number, $n$, of freeenergy minima in series, with $n \in\{5,9,12,16\}$, with measurements of the exit time of an individual walker and experimental tests for the prospect of transport bias. Hence, below we consider a small system of sites, with both unbiased and biased transport, with a particular emphasis on the time a molecular walker takes to exit, which presents scales that are outside the scope of the thermodynamic limit as well as the approximations of Bressloff and others $[8,20]$ discussed above.

Finally, note that the strategic objective is to illustrate how tools from quantum theory can be exploited in stochastic calculations, and in particular how inheriting the excluding properties of Fermions in quantum physics, whereby particles cannot occupy the same state, enables analytical tools, together with symbolic and numerical linear algebra, to be brought to bear on investigating excluded stochastic dynamics in biology and chemistry without the need for ensemble simulations.

In section II below, we detail how the diffusion process maps onto a Schrödinger equation and Fock space, followed in section III with an exploration of Bosonic diffusion without exclusion volume effects. This is generalised in section IV to address volume exclusion effects and Fermion statistics simply by replacing commutation relations in the Fock space by anti-commutation, thus providing a convenient framework for investigating stochastic excluded diffusion, at least for small systems. In section $\mathrm{V}$ we detail a consideration of the mean exit time in the Fock space formalism and compare results between excluded and non-excluded diffusion, with conclusions presented in Section VI.

\section{DIFFUSION PROCESSES IN A LINEAR CHAIN}

In this section we develop a general scheme that enables us to study a classical diffusion process in a linear chain, mapping the governing master equation into a Schrödinger equation both with and without volume exclusion, which corresponds to Fermionic and Bosonic algebras, respectively

First, we consider the chemical master equation that governs the temporal evolution of the probability density $\mathcal{P}(\eta, t)$ associated with finding a fixed number of $N$ particles diffusing in $k$ boxes, with a configuration $\eta=\left\{n_{1}, n_{2}, \ldots n_{k}\right\}$ at time $t$,

$$
\frac{\partial \mathcal{P}(\eta, t)}{\partial t}=\sum_{\eta^{\prime}} T_{\eta^{\prime} \rightarrow \eta} \mathcal{P}\left(\eta^{\prime}, t\right)-T_{\eta \rightarrow \eta^{\prime}} \mathcal{P}(\eta, t),
$$

where

$$
\sum_{j=1}^{k} n_{j}=N
$$

is the total number of particles in all boxes in the chain, and $T_{\eta^{\prime}(\eta) \rightarrow \eta\left(\eta^{\prime}\right)}$ is the transition rate between the configurations $\eta^{\prime}(\eta)$ and $\eta\left(\eta^{\prime}\right)$. The first term on the right of Eqn. 1 takes into account all possible system configurations $\eta^{\prime}=\left\{n_{1}^{\prime}, n_{2}^{\prime}, \ldots n_{k}^{\prime}\right\}$ jumping to configuration $\eta$. 


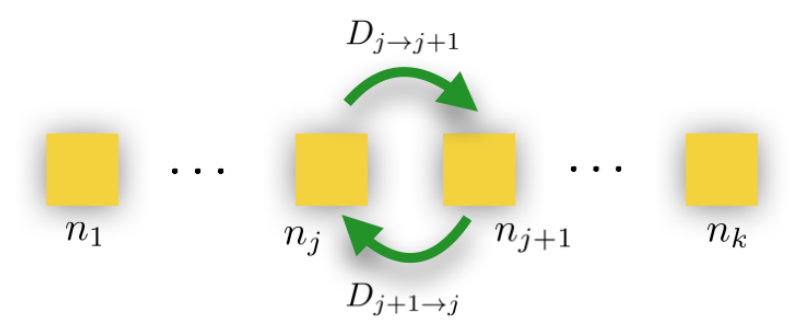

FIG. 1. (Color online) Schematic representation of a $k$-box linear chain. The occupation number for the $j$-th box is given by $n_{j}$, and $D_{j \rightarrow j+1}\left(D_{j+1 \rightarrow j}\right)$ is the probability per unit time per particle to jump from box $j$ to $j+1(j+1$ to $j)$. In the initial examples perfect reflection is assumed if a particle transitions off the end of a chain though for exit time problems, we instead treat the $k$-th box as perfectly absorbing while maintaining perfect reflection on the left edge of the chain. Further, note for definiteness, we have the implicit assumptions that the system is always changing state, so that $D_{j \rightarrow j}=0$, and that longer range transitions are not permitted, though generalisations are also readily considered.

Hence, with the model of Fig. 1 and perfectly reflecting conditions at the chain ends, we have

$$
\begin{aligned}
& \sum_{\eta^{\prime}} T_{\eta^{\prime} \rightarrow \eta} \mathcal{P}\left(\eta^{\prime}, t\right)= \\
& \quad \sum_{j=1}^{k-1} D_{j \rightarrow j+1}\left(n_{j}^{\prime}+1\right) \mathcal{P}\left(n_{j}^{\prime}+1, n_{j+1}^{\prime}-1, t\right) \\
& \quad+D_{j+1 \rightarrow j}\left(n_{j+1}^{\prime}+1\right) \mathcal{P}\left(n_{j}^{\prime}-1, n_{j+1}^{\prime}+1, t\right),
\end{aligned}
$$

where we have used the shorthand notation $\mathcal{P}\left(n_{j} \pm\right.$ $\left.1, n_{j+1} \mp 1, t\right)$ to identify the jumps between neighboring sites at configurations $\eta^{\prime}$ and $\eta$, respectively. In particular, box numbers that do not change are suppressed in this shorthand. The coefficient $D_{j \rightarrow j^{\prime}}$ is defined as the probability per unit time per particle to jump from box $j$ to the box $j^{\prime}$. We can also consider asymmetric diffusion transition rates or cases with $D_{j \rightarrow j} \neq 0$ or different edge conditions or longer range transport in order to keep the process as general as possible, as illustrated below with biased transport, and an absorbing state on the right of the chain once exit times are considered. The second term in Eqn. (1) takes into account all possible departures from configuration $\eta$, given the same assumptions for the first term so that:

$$
\sum_{\eta^{\prime}} T_{\eta \rightarrow \eta^{\prime}} \mathcal{P}(\eta, t)=\sum_{j=1}^{k-1}\left(D_{j \rightarrow j+1} n_{j}+D_{j+1 \rightarrow j} n_{j+1}\right) \mathcal{P}\left(n_{j}, n_{j+1}, t\right)-\gamma\left(D_{j \rightarrow j+1}+D_{j+1 \rightarrow j}\right) n_{j} n_{j+1} \mathcal{P}\left(n_{j}, n_{j+1}, t\right)
$$

In the last term of the above equation we define the volume exclusion parameter $\gamma \in\{0,1\}$, which is zero when there is no limit in the occupation number of the sites (and thus will map onto Bosonic diffusion), and is unity when the occupation number in each site is limited to one particle (and thus will map onto Fermionic diffusion). By considering this term, we eliminate all transitions which are not allowed by volume exclusion [33]. In Appendix A we briefly present a simple example where only three boxes and two particles are considered, and volume exclusion is taken into account, to illustrate the chemical master equation in detail for volume exclusion and Fermions.

To proceed, a given configuration of the system is characterized in Fock space by a direct product of Hilbert spaces $\mathcal{B}_{j}$ for each box in the chain, with $\mathcal{B}_{j} \in$ $\left\{0,1, \ldots, N_{\max }\right\}$, where $N_{\max }$ is the maximum occupation number in the system; hence $N_{\max }=1$ with volume exclusion and is the number of particles otherwise. We thus have $\mathcal{F}=\mathcal{B}_{1} \otimes \ldots \otimes \mathcal{B}_{k}$. The Dirac representation then emerges as follows: let $\left|n_{1} \ldots n_{k}\right\rangle$ be denoted as a pure Fock state, with the set of all pure Fock states providing a basis for the Fock space representation of the diffusion process. Then, the probability of being in an arbitrary state $\eta$ at time $t, \mathcal{P}(\eta, t)$, now written as $P_{\eta}(t)$, can be fully characterized by the wave function $|\Psi(t)\rangle$, which is defined via the linear summation of pure Fock states,

$$
|\Psi(t)\rangle:=\sum_{\eta} P_{\eta}(t)|\eta\rangle
$$

which is analogous to the definition in quantum mechanics. However here, with stochastic mechanics, the coefficients are probabilities with no concept of phase, whereas in quantum mechanics the coefficients are complex, with the squared amplitude representing probability, while possessing an additional phase.

We introduce the creation and annihilation ladder operators for the $j$-th box of the chain, which act on the pure Fock states via

$$
\begin{aligned}
\alpha_{j}^{\dagger}|\eta\rangle & =\left|n_{1} \ldots\left(n_{j}+1\right) \ldots n_{k}\right\rangle \\
\alpha_{j}|\eta\rangle & =n_{j}\left|n_{1} \ldots\left(n_{j}-1\right) \ldots n_{k}\right\rangle,
\end{aligned}
$$

with linearity determining how $\alpha_{j}^{\dagger}$, and $\alpha_{j}$ operate on a general element of the Fock space. It is easy to check that for a Bosonic system these operators satisfy 
the commutation rule $\left[\alpha_{i}, \alpha_{j}^{\dagger}\right]=\delta_{i j}$, while in contrast for the Fermionic system we have the analogous anticommutation rule $\left\{\alpha_{i}, \alpha_{j}^{\dagger}\right\}=\delta_{i j}$. It is worth noting that creation and annihilation ladder operators defined in Eqn. (4), have different weightings compared to those defined in quantum mechanics, in order to produce the correct multiplicity of each classical hop transition between neighbouring sites [22]. Hence, given $n$ particles in a specific site of the chain and the application of the creation ladder operator on $|n\rangle$, another particle is created, and there is an only way to do this; hence $\alpha^{\dagger}|n\rangle=|n+1\rangle$. Conversely, application of the annihilation ladder operator to the same $|n\rangle$ gives $n$ different ways to retrieve a particle, so that $\alpha|n\rangle=n|n-1\rangle$.

Hence the chemical master equation of Eqn. (1) can be recast as a Schrödinger equation, with $i \hbar=1$, via

$$
\frac{\partial|\Psi(t)\rangle}{\partial t}=-H_{\gamma}\left(\alpha_{1}^{\dagger}, \alpha_{1}, \ldots, \alpha_{k}^{\dagger}, \alpha_{k}\right)|\Psi(t)\rangle,
$$

where, for the same assumptions as Eqn. (2),

$$
H_{\gamma}=-\sum_{j=1}^{k-1}\left(D_{j \rightarrow j+1} h_{j \rightarrow j+1}^{\gamma}+D_{j+1 \rightarrow j} h_{j+1 \rightarrow j}^{\gamma}\right),
$$

with

$$
h_{j \rightarrow j^{\prime}}^{\gamma}=\left(\alpha_{j^{\prime}}^{\dagger}-\alpha_{j}^{\dagger}\right) \alpha_{j}+\gamma \alpha_{j}^{\dagger} \alpha_{j} \alpha_{j^{\prime}}^{\dagger} \alpha_{j^{\prime}} .
$$

The resulting solution of the above Schrödinger equation is then explicitly given by

$$
|\Psi(t)\rangle=\exp \left(-H_{\gamma}\left(\alpha_{1}^{\dagger}, \alpha_{1}, \ldots, \alpha_{k}^{\dagger}, \alpha_{k}\right) t\right)|\Psi(0)\rangle .
$$

In addition, for a homogeneous and unbiased diffusion process, so that $D_{j \rightarrow j+1}=D_{j+1 \rightarrow j} \equiv D$, the system simplifies, with the quasi-Hamiltonian of Eqn. (6) reducing to

$$
\begin{aligned}
& H_{\gamma}=D \sum_{j=1}^{k-1}\left(\alpha_{j+1}^{\dagger}-\alpha_{j}^{\dagger}\right)\left(\alpha_{j+1}-\alpha_{j}\right) \\
& -2 \gamma D \sum_{j=1}^{k-1} \alpha_{j}^{\dagger} \alpha_{j} \alpha_{j+1}^{\dagger} \alpha_{j+1} .
\end{aligned}
$$

A fundamental aspect of this formalism is the matrix representation for $H_{\gamma}$ with respect to a basis of pure Fock states, though its determination is analytically feasible only for small systems; more generally $H_{\gamma}$ can be readily found by symbolic computation using the Maple code in the Supplementary Material. Once a matrix representation of $H_{\gamma}$ is found, the calculation of $\exp \left(-H_{\gamma} t\right)$ can be achieved using a variety of available linear algebra methods [34], in turn allowing the calculation of the time-dependent Fock-space state representing the system in terms of the initial state.

In particular, this process can typically be simplified using the Jordan normal form of the quasiHamiltonian, $J_{H_{\gamma}}=Q^{-1} H_{\gamma} Q$, so that $|\Psi(t)\rangle=$
$Q \exp \left(-J_{H_{\gamma}} t\right) Q^{-1}|\Psi(0)\rangle$ and, more generally, one can numerically compute the exponential $\exp \left(-H_{\gamma} t\right)$ at required timepoints. Furthermore, in cases, such as here, with the absence of non-nearest neighbour transitions we can take advantage of the sparsity of the matrix representing $H_{\gamma}$ for numerical linear algebra to reduce the number of floating point operations and memory requirements for larger systems. We also note that once the system is large enough to become a genuine computational challenge, the methodology presented here is no longer appropriate. Instead the system is then large enough to utilise the established van Kampen perturbative expansion of the chemical master equation with respect to the reciprocal of the square root of the system size, which generates the thermodynamic limit of the system and, if required, leading order stochastic corrections [35].

In the following section, we apply the general framework developed here to study the diffusion of Bosonic, and Fermionic particles on a chain lattice. First, we will analytically diagonalize the system for small lattices $(2$ or 3 boxes), with a small number of particles so that we can consider the effect of volume exclusion. Further, for a chain lattice with arbitrary number of boxes, we will find a matrix representation of the system which, consequently, gives us all its information apart from computing $\exp \left(-H_{\gamma} t\right)$ for the Bosonic, and Fermionic cases by simply using the symbolic Maple code in the Supplementary Material.

\section{BOSONIC DIFFUSION ON A LINEAR CHAIN}

To examine the impact of volume exclusion for diffusion on a linear chain we must first consider its absence and thus we examine Bosonic diffusion. While particularly simple, we initially restrict ourselves to Bosonic diffusion on linear chains with two or three boxes, both for reasons of pedagogy and for the provision of the simplest baseline in the form of the three box bosonic diffusion case, from which we can proceed to examine the effect of volume exclusion. We further study larger box numbers and biased diffusion, comparing and contrasting analyses with and without volume exclusion. In addition, while not the focus of this manuscript, there are numerous further non-trivial, but tractable, generalisations of bosonic diffusion that can be explored with the baseline developed in this framework, such as the impact of reactions.

\section{A. Two Boxes and two Particles}

We start our analysis by considering the simplest case of two particles in two boxes with reflecting boundaries at the end of the chain. Three states are available given the absence of volume exclusion: $|1\rangle=|20\rangle,|2\rangle=|11\rangle$, and $|3\rangle=|02\rangle$ and the matrix representation of the quasiHamiltonian in this case is a simple $3 \times 3$ centrosymmetric 
matrix:

$$
H_{0}=\left(\begin{array}{ccc}
2 D & -D & 0 \\
-2 D & 2 D & -2 D \\
0 & -D & 2 D
\end{array}\right)
$$

The general solution is given by the exponential of the Hamiltoniam, as in Eqn. (8), which can be found through the spectra of $H_{0}$. The characteristic polynomial of $H_{0}$ is

$$
p(\lambda)=(2 D-\lambda)\left((2 D-\lambda)^{2}-4 D^{2}\right),
$$

giving us the eigenvalues $\lambda=\{0,2 D, 4 D\}$. Using the Jordan normal form we can evaluate $e^{-H_{0} t}$ by writing $H_{0}=Q J_{\mathrm{H}_{0}} Q^{-1}$. Therefore, we have $|\Psi(t)\rangle=$ $Q e^{-J_{\mathrm{H}_{0} t} t} Q^{-1}|\Psi(0)\rangle$, where

$$
e^{-J_{\mathrm{H}_{0}} t}=\left(\begin{array}{ccc}
1 & 0 & 0 \\
0 & e^{-2 D t} & 0 \\
0 & 0 & e^{-4 D t}
\end{array}\right)
$$

with

$$
Q=\left(\begin{array}{ccc}
1 & 1 & 1 \\
2 & 0 & -2 \\
1 & -1 & 1
\end{array}\right)
$$

and finally we can formally write our solution to $|\Psi(t)\rangle$ as linear combination of the pure Fock states

$$
|\Psi(t)\rangle=P_{1}(t)|1\rangle+P_{2}(t)|2\rangle+P_{3}(t)|3\rangle .
$$

By choosing $|\Psi(0)\rangle=|1\rangle$, these probabilities are

$$
\begin{aligned}
& P_{1}(t)=\frac{1}{4}+\frac{1}{2} e^{-2 D t}+\frac{1}{4} e^{-4 D t}, \\
& P_{2}(t)=\frac{1}{2}-\frac{1}{2} e^{-4 D t}, \\
& P_{3}(t)=\frac{1}{4}-\frac{1}{2} e^{-2 D t}+\frac{1}{4} e^{-4 D t} .
\end{aligned}
$$

In Fig. 2 we illustrate the time evolution of the probabilities of finding the system in one of three configurations 15(a)-15(c). The equilibrium probabilities, i.e. the probabilities in the limit $t \rightarrow \infty$ are not equal, as one might expect from detailed balance on noting $|2\rangle=|11\rangle$ has multiplicity two, as the particles are distinguished.

\section{B. Three boxes and two particles}

Since the Fermionic case with two boxes and particles is trivial, we proceed to examine two particles diffusing in three boxes with reflecting boundary conditions and consider the impact of volume exclusion. In

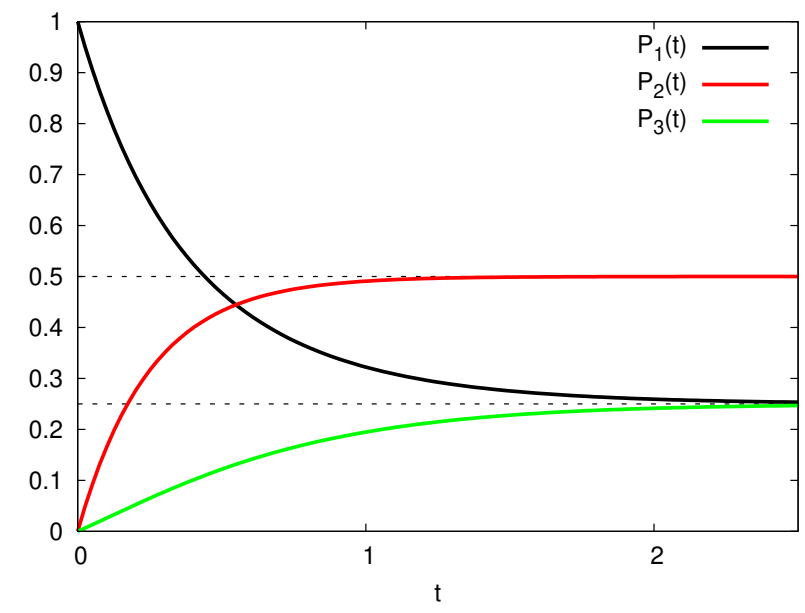

FIG. 2. (Color online) Two Bosonic particles diffusing in a two box chain with reflecting boundary conditions: temporal evolution of the probability of finding the system in a state $\eta, P_{\eta}(t)$. Here, $|\Psi(0)\rangle=|20\rangle$ and $D=1$ where, without loss of generality and as throughout this paper, the time unit has been subject to non-dimensionalisation. The horizontal dotted lines represent the equilibrium values of these quantities: $P_{1}(t \rightarrow \infty)=P_{3}(t \rightarrow \infty)=1 / 4$, and $P_{2}(t \rightarrow \infty)=1 / 2$.

the Bosonic case, with the absence of exclusion, we have six possible states for the base set: $|1\rangle=|200\rangle$, $|2\rangle=|110\rangle,|3\rangle=|101\rangle,|4\rangle=|020\rangle,|5\rangle=|011\rangle$, and $|6\rangle=|002\rangle$. By diagonalizing the matrix representation of the quasi-Hamiltonian (see Supplementary Material for an explicit expression of the matrix) we find the eigenvalues $\lambda=\{0, D, 2 D, 3 D, 4 D, 6 D\}$, and therefore the probabilities $P_{\eta}(t)$ for each state in the base set, with $\eta \in\{|1\rangle, \ldots,|6\rangle\}$, analogously to the previous subsection. In calculating statistical quantities that depend on higher moments, such as the variance, it will be useful to consider the $l$-th moment of the average occupation number in the $j$-th box,

$$
\left\langle n_{j}^{l}(t)\right\rangle=\sum_{\eta}\left(b_{j}^{\eta}\right)^{l} P_{\eta}(t) ; \quad l \in \mathbb{N}
$$

with $b_{j}^{\eta}$ denoting the number of particles in the $j$-th box given that the system is in the $\eta$ state.

In Fig. 3 we plot the mean occupation number, $\left\langle n_{j}\right\rangle$, as a function of time, together with the variance

$$
\sigma_{j}^{2}(t)=\left\langle\left(n_{j}(t)-\left\langle n_{j}(t)\right\rangle\right)^{2}\right\rangle=\left\langle n_{j}^{2}\right\rangle-\left(\left\langle n_{j}\right\rangle\right)^{2},
$$

computed via Eqn. (16), as function of the time. These two quantities reach their equilibrium values, represented by dotted lines in Fig. 3 , at $\left\langle n_{j}(t \gg 1)\right\rangle \equiv\langle n\rangle=2 / 3$, and $\sigma_{j}^{2}(t \gg 1) \equiv \sigma^{2}=4 / 9$, for large times, respectively.

Note that the particles are not distinguished for this observable and that at large time for the equilibrium distribution, the effects of not losing a particle to the left of the left most box cancels the effects of a particle never entering from the left, and similarly for the very rightmost 
box. Hence all boxes are equivalent at large time and thus one has the $l$-th moment for the large time asymptote of the occupation number, $n$, for each box can be deduced from the simple combinatorial problem of arranging $N_{\mathrm{p}}$ particles in $k$ boxes, so that

$$
\left\langle n^{l}\right\rangle=\sum_{n=0}^{N_{\mathrm{p}}}\left(\begin{array}{c}
N_{p} \\
n
\end{array}\right) \frac{n^{l}(k-1)^{N_{\mathrm{p}}-n}}{k^{N_{\mathrm{p}}}},
$$

which, on evaluation, is in agreement with Fig. 3.

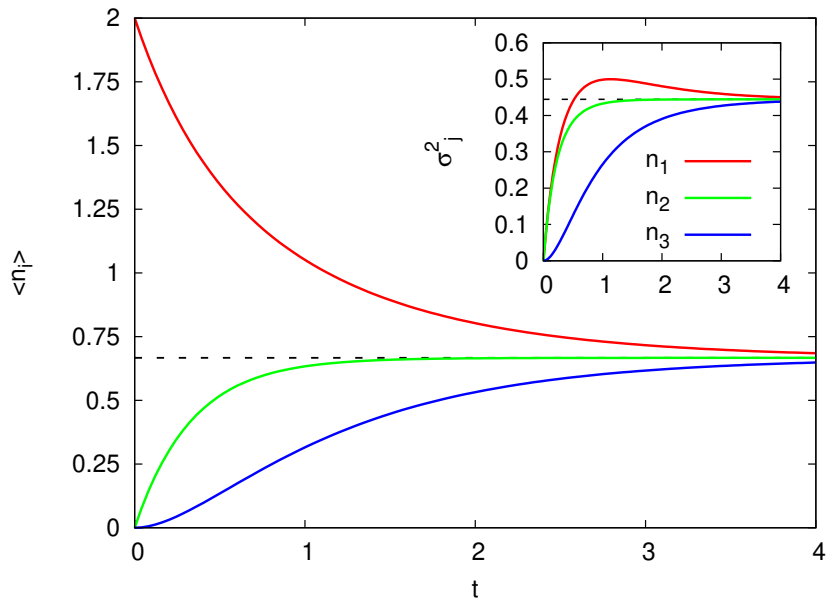

FIG. 3. (Color online) Bosonic diffusion with particles in three boxes: the temporal evolution of the mean occupation number, $\left\langle n_{j}\right\rangle$, and variance $\sigma_{j}^{2}$ (inset), in the $j$-th box $(j=$ $1,2,3)$. Here, $|\Psi(0)\rangle=|200\rangle$ and $D=1$.

\section{General case}

Since there is no volume exclusion, one can treat Bosons as uncorrelated particles, so the system's configuration in a time $t$ can be written as follows

$$
|\Psi(t)\rangle=\left|\psi_{1}(t)\right\rangle \otimes \cdots \otimes\left|\psi_{N_{\mathrm{p}}}(t)\right\rangle,
$$

where, $\left|\psi_{i}(t)\right\rangle=\exp \left\{-H_{0} t\right\}\left|\psi_{i}(0)\right\rangle$, with $\left|\psi_{i}(0)\right\rangle$ the initial condition of the $i$-th particle in one of the $k$ boxes of the chain.

A straightforward exemplar of the uncorrelated behavior of Bosons is to recover the basis set for the two boxes and two particles case, as discussed in subsection III A, from the two boxes and a single particle one. The diffusion of one particle in two box provides only two possible states: $\left|1^{\prime}\right\rangle=|10\rangle$, and $\left|2^{\prime}\right\rangle=|01\rangle$. The probabilities of finding the system in one of these states, given that the particles is initially in the states $\left|1^{\prime}\right\rangle$ and $\left|2^{\prime}\right\rangle$, are $P_{1}^{\prime}(t)=\left(1+e^{-2 D t}\right) / 2$ and $P_{2}^{\prime}(t)=\left(1-e^{-2 D t}\right) / 2$, respectively. The probabilities given by Eqn. (15) can be obtained from $P_{1,2}^{\prime}(t)$ through: $P_{1}(t)=P_{1}^{\prime 2}(t)$, $P_{2}(t)=2 P_{1}^{\prime}(t) P_{2}^{\prime}(t)$ and $P_{3}(t)=P_{2}^{\prime 2}(t)$, respectively.
Let us consider for example a ten boxes chain with two particles. In this situation we have 55 possible configurations available for the system, since the number of configurations for $N_{\mathrm{p}}$ particles and $N$ boxes is given by the binomial coefficient

$$
\left(\begin{array}{c}
N_{\mathrm{p}}+N-1 \\
N-1
\end{array}\right)
$$

which concurs with the multiplicity of the associated Einstein Solid. In turn, we generate a $55 \times 55$ matrix for the quasi-Hamiltonian, which is relatively demanding symbolically if we wish to analytically find the spectrum of the Hamiltonian. Nevertheless, using the fact that the particles are uncorrelated, one only needs to deal with a $10 \times 10$ quasi-Hamiltonian related with the diffusion of a single particle in the chain. The combined probability of finding the system in one of the 55 configurations is simply given by the product of the probabilities associated with the single particle situation, together with an appropriate binomial coefficient.

In Fig. 4 we show the mean occupation number, and the variance (in the inset) of each box as function of time. Both particles are in the leftmost box initially and therefore the initial condition of the combined system is given by Eqn. (18), $|\Psi(0)\rangle=\left|\psi_{1}(0)\right\rangle \otimes\left|\psi_{2}(0)\right\rangle=|20 \ldots 0\rangle$. As expected, for the large time regime the mean occupation number tends to $\left\langle n_{j}(t \gg 1)\right\rangle=\langle n\rangle=1 / 5$, and the variance in this regime tends to $\sigma_{j}^{2}(t \gg 1)=\sigma^{2}=9 / 50$. Both results are easily verified from Eqn. (17).

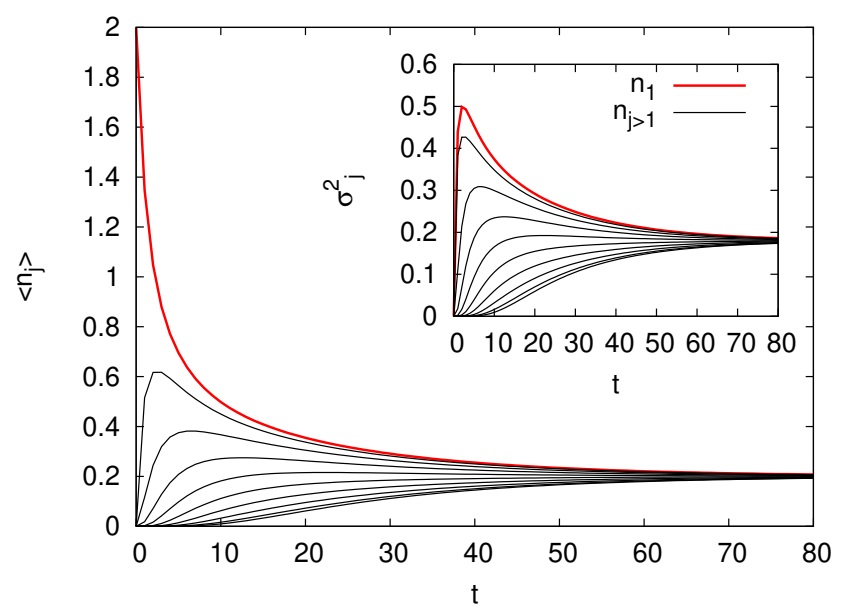

FIG. 4. (Color online) Two Bosonic particles diffusing in ten boxes: temporal evolution of the mean occupation number, $\left\langle n_{j}\right\rangle$, and variance $\sigma_{j}^{2}$ (inset), in the $j$-th box $(j=1,2, \ldots, 10)$. Here, $|\Psi(0)\rangle=|20 \ldots 0\rangle$ and $D=1 / 2$. The highest curve represents the results for box 1 , the second highest curve the results for box 2 and so on. 


\section{FERMIONIC DIFFUSION ON A LINEAR CHAIN}

After these introductory exemplars, we proceed to address volume exclusion effects for diffusion on a linear chain, and thus single file diffusion, by using Fermionic algebra with the Fock space approach. This technique has been considered in the context of binary degradation reactions on a linear ring [33] and in the use of Bethe ansatz solutions for asymmetric exclusion processes on a infinite chain [36], in contrast to the small chains studies of this paper. In particular the restriction to small chains prevents the use of common thermodynamic approximations such as the van Kampen expansions or concepts from the study of Tonk's gas [35, 37, 38].

To proceed, we first note the case with two boxes and two particles is trivial, and hence we start with three boxes and two particles. Later, we will display results for an arbitrary finite number of boxes $k$ and up to $k-1$ Fermions. Once we implement Fermionic diffusion symbolically, we are able to analyze the effect of volume exclusion for unbiased diffusion and the first exit times, as illustrated below.

\section{A. Three boxes and two particles}

With two particles in a chain of three boxes, we have only three possible states, $|1\rangle=|110\rangle,|2\rangle=|101\rangle$, and $|3\rangle=|011\rangle$, with the quasi-Hamiltonian, $H_{1}$, given by:

$$
H_{1}=\left(\begin{array}{ccc}
D & -D & 0 \\
-D & 2 D & -D \\
0 & -D & D
\end{array}\right) .
$$

The general solution for the time evolution of the system is given by Eqn. (8), where the characteristic polynomial of $H_{1}$ is given by

$$
p(\lambda)=(D-\lambda)\left[(D-\lambda)(2 D-\lambda)-2 D^{2}\right] .
$$

The eigenvalues are thus $\lambda=\{0, D, 3 D\}$. Following the same steps described in the previous section one can write $|\Psi(t)\rangle=Q e^{-J_{\mathrm{H}_{1}} t} Q^{-1}|\Psi(0)\rangle$, where

$$
e^{-J_{\mathrm{H}_{1}} t}=\left(\begin{array}{ccc}
1 & 0 & 0 \\
0 & e^{-D t} & 0 \\
0 & 0 & e^{-3 D t}
\end{array}\right),
$$

with

$$
Q=\left(\begin{array}{ccc}
1 & -1 & 1 \\
1 & 0 & -2 \\
1 & 1 & 1
\end{array}\right)
$$

which enables us to determine

$$
|\Psi(t)\rangle=P_{1}(t)|1\rangle+P_{2}(t)|2\rangle+P_{3}(t)|3\rangle .
$$

By choosing $|\Psi(0)\rangle=|1\rangle$, these probabilities reduce to:

$$
\begin{aligned}
& P_{1}(t)=\frac{1}{3}+\frac{1}{2} e^{-D t}+\frac{1}{6} e^{-3 D t}, \\
& P_{2}(t)=\frac{1}{3}-\frac{1}{3} e^{-3 D t} \\
& P_{3}(t)=\frac{1}{3}-\frac{1}{2} e^{-D t}+\frac{1}{6} e^{-3 D t} .
\end{aligned}
$$

Since $b_{j}^{\eta} \in\{0,1\}$, in Eqn. (16), we have that $\left\langle n_{j}^{l}(t)\right\rangle=$ $\left\langle n_{j}(t)\right\rangle$, and thus the variance in the Fermionic case is $\sigma_{j}^{2}(t)=\left\langle n_{j}(t)\right\rangle\left(1-\left\langle n_{j}(t)\right\rangle\right)$, while the averages are easily obtained from Eqn. (23)

$$
\begin{aligned}
& \left\langle n_{1}(t)\right\rangle=P_{1}(t)+P_{2}(t), \\
& \left\langle n_{2}(t)\right\rangle=P_{1}(t)+P_{3}(t), \\
& \left\langle n_{3}(t)\right\rangle=P_{2}(t)+P_{3}(t) .
\end{aligned}
$$

In Fig. 5 we show the temporal evolution of the mean occupation number, $\left\langle n_{j}(t)\right\rangle$, and variance $\sigma_{j}^{2}(t)$, for this small chain of $k=3$ boxes and $N_{\mathrm{p}}=2$ Fermions. While the transient differs from the Bosonic case illustrated in Fig. 3, the equilibrium mean occupation number is the same as the nonexclusive case, with

$$
\left\langle n_{j}(t \gg 1)\right\rangle=\langle n\rangle \equiv N_{\mathrm{p}} / k=2 / 3 .
$$

However the variance is a halved with $\sigma_{j}^{2}(t \gg 1)=$ $\langle n\rangle(1-\langle n\rangle)=2 / 9$.

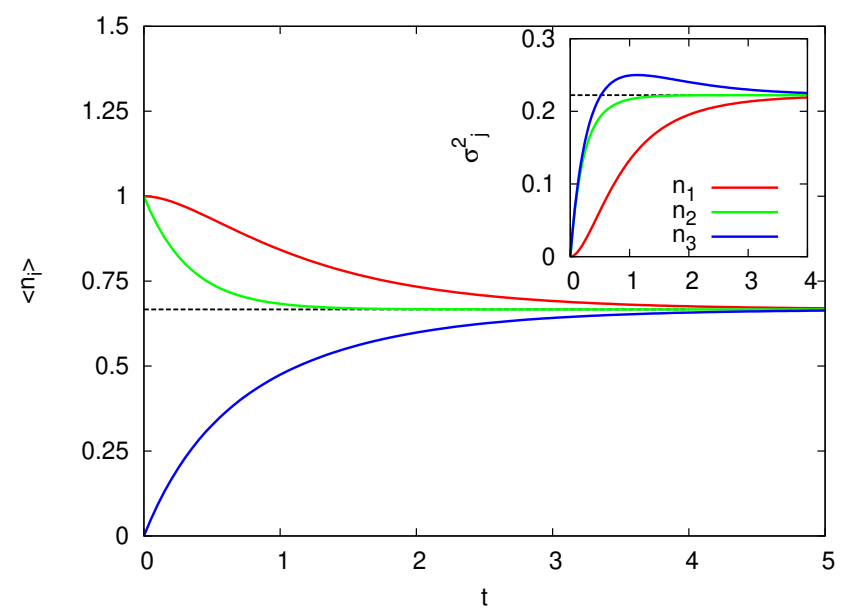

FIG. 5. (Color online) Fermionic diffusion of 2 particles in 3 boxes: temporal evolution of the mean occupation number, $\left\langle n_{j}\right\rangle$, and variance $\sigma_{j}^{2}$, in the $j$-th box $(j=1,2,3)$. Here, $|\psi(0)\rangle=|110\rangle$ and, with a suitable scaling of the time unit, $D=1$. 


\section{B. General case}

Here we want to investigate volume exclusion effects with an arbitrary number of $k$ boxes and $N_{p}<k$ particles. Subject only to computational resources, the Maple code in the Supplementary Material provides a complete characterization of the system. First, note the interesting two-state symmetry between $k$ boxes and $N_{\text {p }}$ particles on

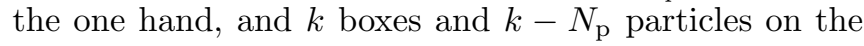
other. This arises as both cases have the same number of allowed configurations, given by the binomial coefficient $k ! / N_{\mathrm{p}} !\left(k-N_{\mathrm{p}}\right) !$. For example, the long-time average mean occupation number of one situation, $n^{\prime}$, can be simply obtained directly from its complement $n$, via $n^{\prime}=1-n$. Hence mapping between diluted and crowded systems can be useful in inferring the impact of diffusion in the crowded scenario with volume exclusion, from the dilute case.

In the Maple code in the supplementary material, we can symbolically implement a Fermionic system with a finite, but otherwise arbitrary, number of boxes. In Fig. 6 we give an illustration of this code by plotting the temporal evolution of the mean occupation number $\left\langle n_{j}\right\rangle$, and variance $\sigma_{j}^{2}$ (inset), for a system with ten boxes and two Fermions. We choose as the initial system's initial state, $|\Psi(0)\rangle=|110 \ldots 0\rangle$. The asymptotic values for large time are $\langle n\rangle=1 / 5$, as we would expect, and the variance is given by $\sigma^{2}=4 / 25$, which is once more smaller than the Bosonic counterpart.

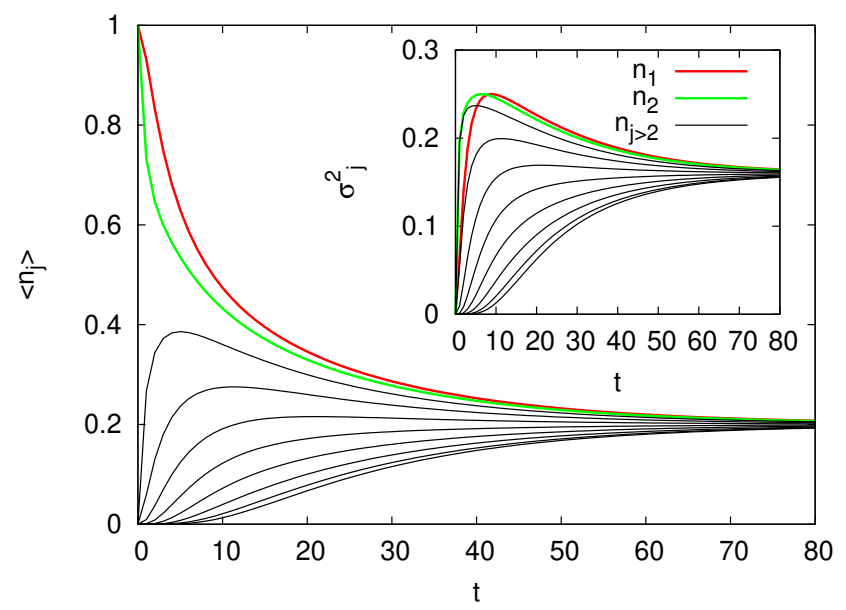

FIG. 6. (Color online) Fermionic diffusion in ten boxes: temporal evolution of the mean occupation number, $\left\langle n_{j}\right\rangle$, and variance $\sigma_{j}^{2}$ (inset), in the $j$-th box $(j=1,2, \ldots, 10)$. Here, $|\Psi(0)\rangle=|110 \ldots 0\rangle$ and $D=1 / 2$. The highest curve represents the results for box 1 , the second highest curve the results for box 2 and so on.

\section{FIRST EXIT TIME}

The analysis of first exit times for random walks has a long history, as well as retaining core importance in modern studies, since the exit time in stochastic systems determines many diverse phenomena, ranging from the timing of a gambler's ruin [1] to shuttling rates in modern prototypical molecular machines [9]. To proceed, we first define the first exit time as the random variable given by the time for one of the particles to arrive at the rightmost box of the linear chain, as highlighted in Fig. 7 where we depict a $k$-box linear chain, with each box occupied by $n_{j}$ particles. A fundamental difference of the system we now investigate to consider first exit times is that now the $k$-th, rightmost box is an absorbing boundary, i.e., once a particle reaches the $\mathrm{k}$-th box it leaves the system and it cannot diffuse back to the left. We also assume that all diffusion transition rates are the same in the same direction, so that $D_{j \rightarrow j+1} \equiv D_{\mathrm{R}}$ and $D_{j+1 \rightarrow j} \equiv D_{\mathrm{L}}$, for all $j$, which allows the prospect of a transport bias in the studies below, as motivated by molecular shuttles [9].

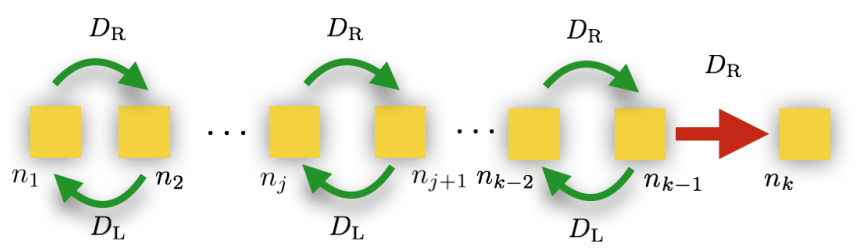

FIG. 7. (Color online) Schematic representation of a $k$-box linear chain with an absorbing box. The occupation number for the $j$-th box is given by $n_{j}$, and $D_{\mathrm{R}}\left(D_{\mathrm{L}}\right)$ represents the diffusion rate from the right(left). The $k$-th box is absorbing, so the diffusion rate to the left is zero.

Taking into account the absorbing right hand boundary, we remove the term that correspond to left jumps between the $k$ and $k-1$ boxes in the general quasiHamiltonian, Eqn. (6). This generates the quasiHamiltonian

$$
H_{\gamma}^{\prime}=-D_{\mathrm{R}} \sum_{j=1}^{k-1} h_{j \rightarrow j+1}^{\gamma}-D_{\mathrm{L}} \sum_{j=1}^{k-2} h_{j+1 \rightarrow j}^{\gamma},
$$

for finding the mean exit time at an absorbing right-hand boundary. We also define the set of exit states to be $\eta_{\text {exit }}$, where corresponds to at least one particle in the rightmost box.

The probability of finding the system in a non-exit state at time $t$, and thus no particle having left by time $t$, is thus given by

$$
P\left(\eta \notin \eta_{\text {exit }}, t\right)=\sum_{\eta \neq \eta_{\text {exit }}}\left\langle\eta\left|e^{-H_{\gamma}^{\prime} t}\right| \Psi(0)\right\rangle .
$$

We note that symbolically, its calculation is realised via the use of an indicator mapping, which generates unity 
if it acts on a ket, $|i\rangle$, that is both a base and exit state, and gives zero for any base state that is not an exit state.

With $T$ the random variable for the first exit time of any particle, we have the probability that the first exit time is $t$ or less is given by

$$
P(T \leq t)=1-\sum_{\eta \notin \eta_{\text {exit }}}\left\langle\eta\left|e^{-H_{\gamma}^{\prime} t}\right| \Psi(0)\right\rangle=: F(t) .
$$

Hence the probability distribution of the first exit time reads $[8]$

$$
f(t)=\frac{\partial F(t)}{\partial t}=-\frac{\partial}{\partial t} \sum_{\eta \notin \eta_{\text {exit }}}\left\langle\eta\left|e^{-H_{\gamma}^{\prime} t}\right| \Psi(0)\right\rangle,
$$

and all moments of the exit time distribution can be calculated through the following integral

$$
\left\langle T^{l}\right\rangle=\int_{0}^{\infty} t^{l} f(t) d t
$$

We symbolically implemented calculation of the first exit time distribution using the steps demonstrated in this section, as presented in the Maple code within the supplementary material. As further validation of the Maple code, first note that the lattice covering time is defined as the mean time a single Bosonic particle takes to visit all sites of the lattice. Hence, if we consider a single Fermionic or Bosonic particle initially at the leftmost box, $|\Psi(0)\rangle=|10 \ldots 0\rangle$, the lattice covering time is exactly the mean first exit time. In particular, we then find the mean first exit time is equal to $k(k-1)$ by the symbolic evaluation of Eqn. 29 for $l=1$, in agreement with Yokoi et al. [39] where $k(k-1)$ is determined via the lattice covering time of a random walk on a chain lattice with the same reflecting boundary conditions.

In the following subsections we compare the mean first exit time for Bosons and Fermions, to assess how volume exclusion impacts on exit times and, in particular, we examine the extent of the differences between unbiased and biased diffusion processes for different numbers of particles and different chain lengths.

\section{A. Fermion vs. Boson with Unbiased Diffusion on a Linear Chain}

Here we consider unbiased diffusion, so the diffusion rates to left and to the right are equal, so that

$$
D_{\mathrm{R}}=D_{\mathrm{L}}=1 / 2,
$$

where the final equality is, without loss, by choice of timescale. We proceed to analyze a ten box chain with two particles - a dilute scenario - and subsequently an intermediate case of four particles within eight boxes and a final crowded case of with eight particles within ten boxes.

\section{Dilute Particle Levels}

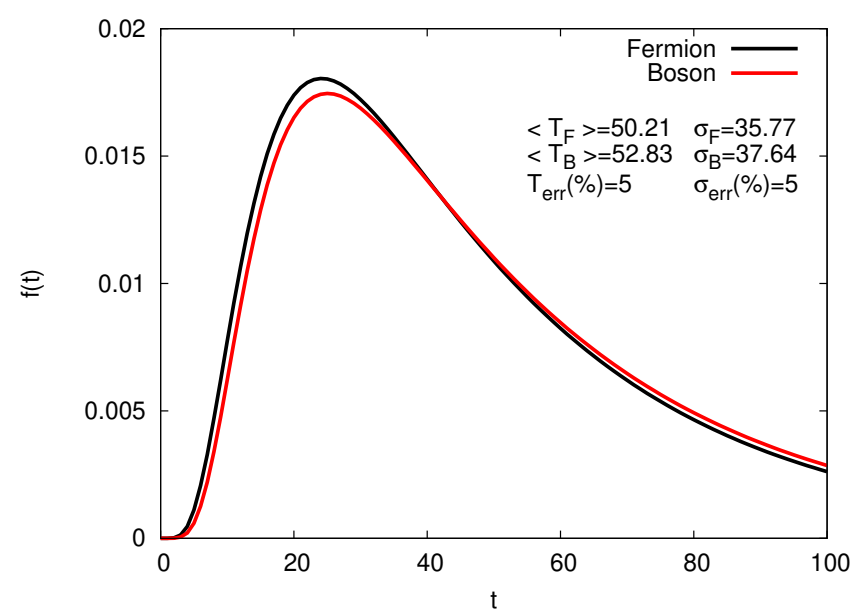

FIG. 8. (Color online) The probability distribution of the exit time for a dilute situation of ten boxes and two particles, given both particles start as far left as allowed. Note that $<T_{\mathrm{B}}>,<T_{\mathrm{F}}>$, are respectively the mean exit time for Bosonic and Fermionic particles, together with the associated standard deviation, $\sigma_{\mathrm{F}}, \sigma_{\mathrm{B}}$. The quantities $T_{\text {err }}$ and $\sigma_{\text {err }}$ respectively measure the mean and standard deviation of the relative difference of the Fermionic and Bosonic distributions, and is defined in the main text.

In Fig. 8 we present the first exit time probability distribution, where particle levels are dilute, with two particle within ten boxes. The initial state has the particles as left as allowed, so that for Fermions, the initial state at $t=0$ is

$$
|\Psi(0)\rangle=|110 \ldots 00\rangle,
$$

while for Bosons

$$
|\Psi(0)\rangle=|200 \ldots 00\rangle .
$$

As we can observe in Figure 8, the Bosonic and Fermionic exit time distributions are very similar and hence the mean first exit times are very close to each other. If the Bosonic initial distribution was set to be $|\Psi(0)\rangle=$ $|110 \ldots 00\rangle$ one would have even closer agreement between the Bosonic and Fermionic cases (not presented), emphasising the intuitive idea that volume exclusion may neglected for sufficiently dilute diffusion. In Fig. 8 the relative error, $T_{\text {err }} \equiv\left|\left\langle T_{\mathrm{F}}\right\rangle-\left\langle T_{\mathrm{B}}\right\rangle\right| /\left\langle T_{\mathrm{F}}\right\rangle$, which quantifies the error on disregarding volume exclusion when calculating the mean first exit time, is given for this exemplar and found to be of the order of $5 \%$. Analogously, the scale of the relative error in the root mean square, $\sigma_{\text {err }} \equiv\left|\sigma_{\mathrm{F}}-\sigma_{\mathrm{B}}\right| / \sigma_{\mathrm{F}}$, is once more approximately $5 \%$.

\section{Intermediate dilution}

We proceed to consider a case of intermediate dilution, where the four particles in the system is half of 
the number of boxes. For Fermions we choose the initial condition $|\Psi(0)\rangle=|11110000\rangle$ and present the first exit time probability distribution in Fig. 9. For Bosons we choose two initial conditions: the first one (solid red line) in similar to the Fermionic case, and the second one (dashed red line) $|\Psi(0)\rangle=|40000000\rangle$. One can observe from Fig. 9, that these different initial conditions produce very different distributions. Comparing the distributions for Fermions, and Bosons with the same initial condition, we observe qualitatively the same behavior, although the relative error for mean first exit time is about $12 \%$. Hence as crowding increases we see the importance of initial conditions and the increased discrepancy introduced by the neglect of volume exclusion.

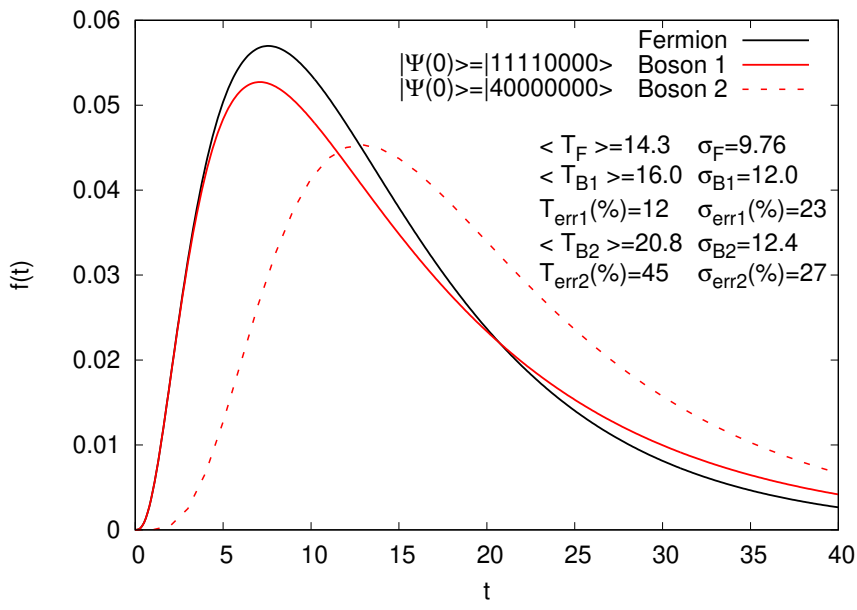

FIG. 9. (Color online) The probability distribution for the exit time with eight boxes and four particles. The Fermionic initial condition is given by $|\Psi(0)\rangle=|11110000\rangle$ and the initial conditions for each of the two Bosonic curves are given in the Figure legend, with the definitions of $\left\langle T_{\mathrm{B} 1}\right\rangle,\left\langle T_{\mathrm{F}}\right\rangle$, $<\sigma_{\mathrm{B} 1}>,<\sigma_{\mathrm{F}}>, T_{\mathrm{err} 1}$ and $\sigma_{\mathrm{err} 1}$ inherited from Figure 8 and the main text of Section V A 1, where the Bosonic summary statistics are for the initial condition of Boson 1, with analogous definitions for Boson 2.

\section{Crowded Particles}

Finally, in Fig. 10 we depict the probability distributions for crowded particles, with 10 boxes and 8 particles. Noting in the previous example that placing all Bosons on the left of the domain leads to significant deviation from the Fermionic case once crowding becomes apparent, an identical initial condition of $|\Psi(0)>=| 1111111100>$ is used for both Bosons and Fermions. For very short times the distribution for Fermions (in black), and Bosons (in red) are very similar, but around and after their maxima they start to differ. Nonetheless, given the same initial conditions one observes that the Fermionic mean first exit time is shorter than the Bosonic first exit time. Furthermore, the relative error in the mean first exit times between the Fermionic and Bosonic computational studies is $25 \%$, while the variance possesses relative errors that are as high as $58 \%$. In turn, this emphasises that a small ensemble of runs would not be sufficient to control for the effects of noise in examining the impact of volume exclusion on small population and stochastic diffusive processes as crowding becomes important.

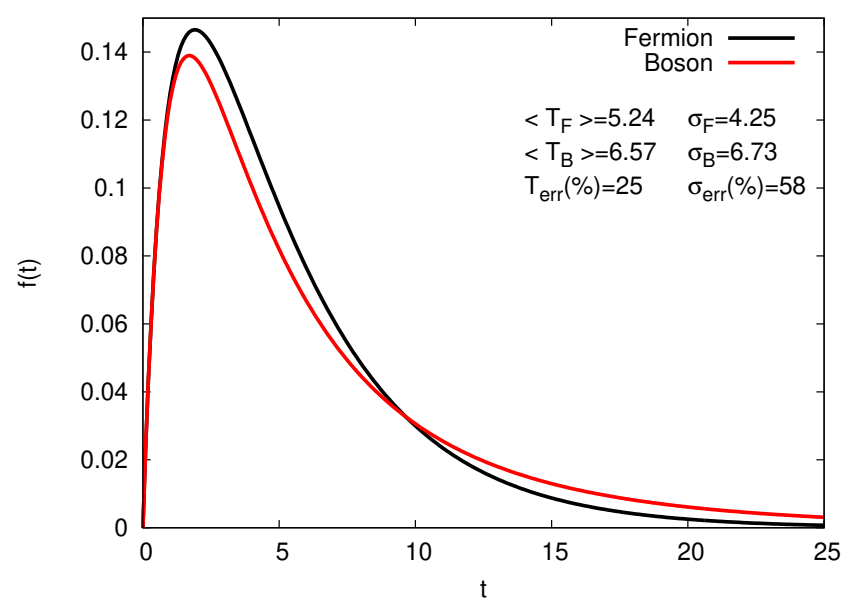

FIG. 10. (Color online) The probability distribution of the exit time in a crowded situation, with ten boxes and eight particles. Here we choose the same initial condition for both cases: $|\Psi(0)\rangle=|111 \ldots 100\rangle$ and the definitions of $\left\langle T_{\mathrm{B}}\right\rangle$, $\left.<T_{\mathrm{F}}\right\rangle,\left\langle\sigma_{\mathrm{F}}\right\rangle,\left\langle\sigma_{\mathrm{B}}\right\rangle, T_{\text {err }}$ and $\sigma_{\text {err }}$ are inherited from Figure 8 and the main text of Section VA 1.

\section{B. Fermion vs. Bosons, with Biased Diffusion on a Linear Chain}

We continue comparing the dynamics of Bosonic and Fermionic transport, generalised to consider biased homogeneous diffusion and how this may influence the impact of volume exclusion on the first exit time. In particular, a bias in diffusive transport to the right is introduced by enforcing diffusion transition rates to the right, $D_{\mathrm{R}}$, larger than those to the left, $D_{\mathrm{L}}$. Our results for an eight box chain and four particles is shown in Fig. 11, where we consider four different values for the diffusion rates (subject to the same temporal non-dimensionalisation): $D_{\mathrm{R}}=0.6, D_{\mathrm{L}}=0.4$ in panel $11(\mathrm{a}), D_{\mathrm{R}}=0.7, D_{\mathrm{L}}=0.3$, in panel $11(\mathrm{~b}), D_{\mathrm{R}}=0.8, D_{\mathrm{L}}=0.2$ in panel $11(\mathrm{c})$, and $D_{\mathrm{R}}=0.9, D_{\mathrm{L}}=0.1$ in panel $11(\mathrm{~d})$. The initial condition for Fermions and Bosons is the same for all these examples and given by $|\Psi(0)\rangle=|11110000\rangle$.

As we can see from the distributions of Fig. 11, as the diffusion rate $D_{\mathrm{R}}$ increases the distribution for Fermions become broader than the Bosonic counterpart. One consequence of this broadening is that the mean first time for Fermions is larger than Bosons, in distinct contrast to observations from unbiased diffusive dynamics. 


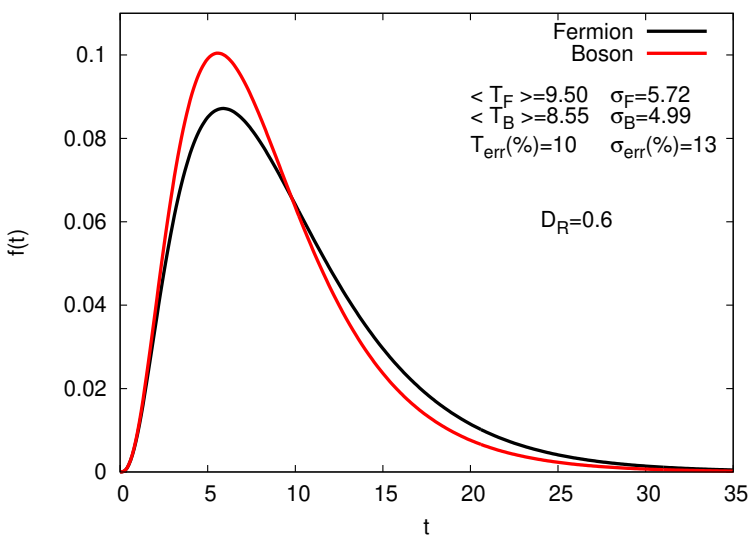

(a)

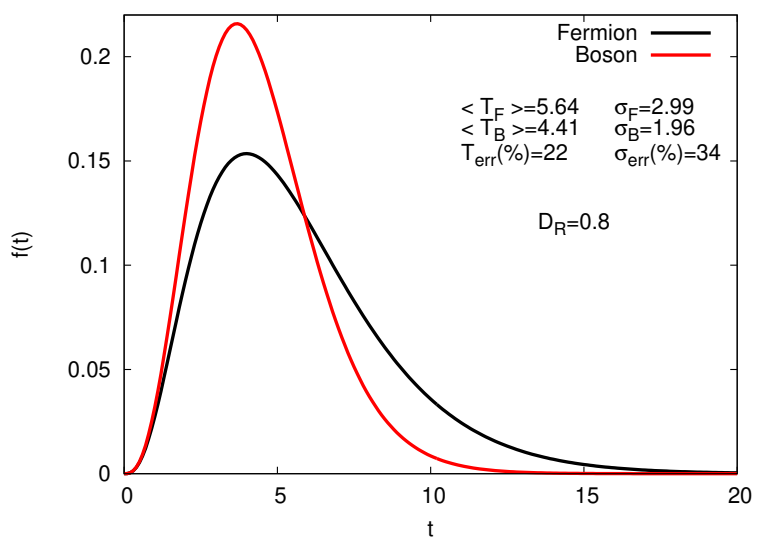

(c)

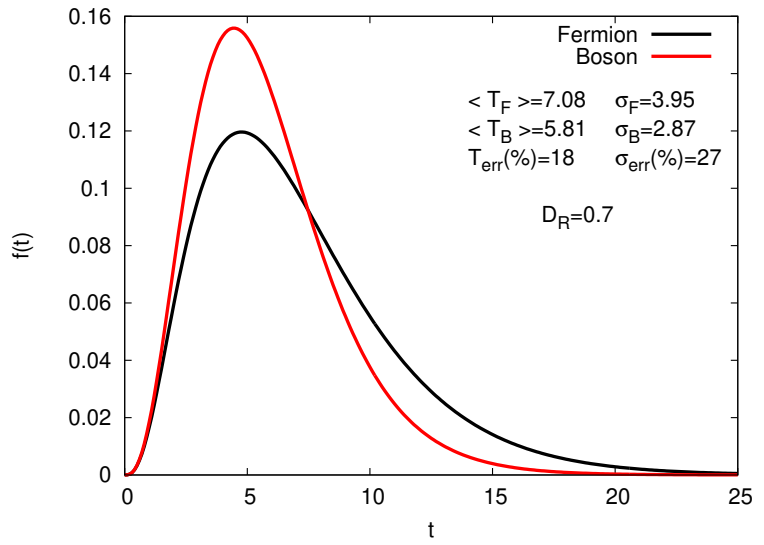

(b)

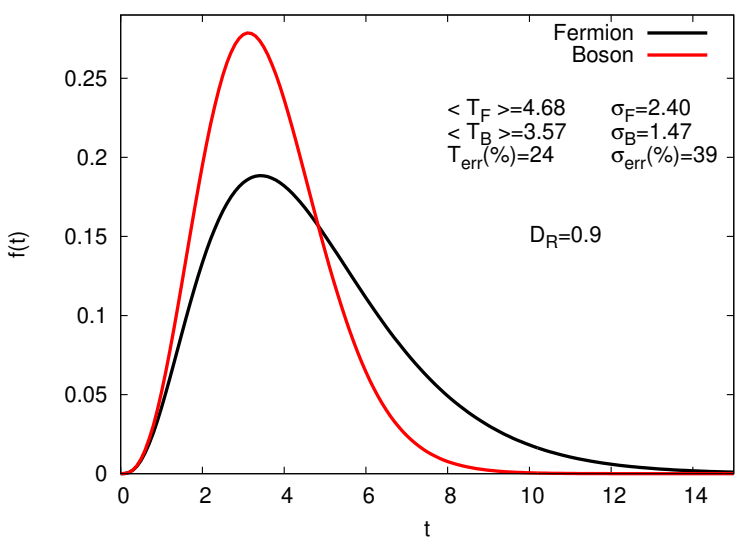

(d)

FIG. 11. (Color online) The first exit time probability distribution for a biased diffusion with four particles in eight boxes. With the scaling temporal non-dimensionalisation throughout, in panel (a) we consider diffusion rates $D_{\mathrm{R}}=0.6, D_{\mathrm{L}}=0.4$, in (b) $D_{\mathrm{R}}=0.7, D_{\mathrm{L}}=0.3$, (c) $D_{\mathrm{R}}=0.8, D_{\mathrm{L}}=0.2$, and (d) $D_{\mathrm{R}}=0.9, D_{\mathrm{R}}=0.1$. The definitions of $\left\langle T_{\mathrm{B}}\right\rangle,\left\langle T_{\mathrm{F}}\right\rangle,\left\langle\sigma_{\mathrm{F}}\right\rangle$, $\left.<\sigma_{\mathrm{B}}\right\rangle, T_{\text {err }}$ and $\sigma_{\text {err }}$ are inherited from Figure 8 and the main text of Section VA 1 .

\section{CONCLUSIONS}

In this work, we have developed a systematic framework for analytical or numerical calculations associated with modelling of transport processes where finite size effects prevent two random walkers occupying the same location. The approach first requires writing the chemical master equation for the diffusion process in terms of creation and annihilation ladder operators. In particular we emphasise that changing from non-exclusive (Bosonic) diffusion to volume-excluded (Fermionic) diffusion corresponds to a change in these operators from standard commutation relations to standard anti-commutation relations, highlighting how Fermi statistics can be used for stochastic systems with excluded states. The methodology then proceeds by generating a matrix representation of the resulting Fock space that, in turn, generates a matrix ordinary differential equation for the stochastic evolution of the system. This differential equation may then be solved by direct numerical methods, or as here, simpli- fied using linear or symbolic algebra, essentially enabling an analytical/symbolic solution for the wavefunction of the system, which encodes all stochastic dynamics without the need for considering stochastic simulation and ensemble statistics.

The methodology is not suited to large systems of particles, due to the curse of dimensionality, but larger systems are very well-suited to the van Kampen expansion and the continuum approximation and thus generally well studied (e.g. $[35,40,41]$ ). In contrast our focus is small systems where stochasticity is important, and we illustrate the methods with a relatively small number of particles and boxes and we have compared and contrasted occupation numbers and exit times for nonexclusive (Bosonic) diffusion and volume-excluded (Fermionic) for single file diffusion.

We initially considered the simplest initial examples to provide a baseline for further study, to illustrate the method and also to allow an element of validation, by confirming agreement with independent calculations of 
steady state distributions for reflecting boundary conditions and lattice covering times in the presence of an absorbing state. We proceeded to consider the exit time problem for single file diffusive processes on the scale of those observed for shuttling in molecular machines [9] with around 10 sites and a range of particle numbers.

Among the first observations of the exit time stochastic dynamics was that at sufficient dilution Fermionic and Bosonic diffusion gave essentially the same predictions for the exit time distributions (and the stochastic dynamics more generally), as intuitively one would expect, which thus provides further validation. However, once crowding starts to emerge the impact of volume exclusion becomes apparent and is more substantial once the particle number is half that of the number of sites, especially if initial states with multiple occupancy are considered for Bosonic diffusion.

We also observed, at least for unbiased transport, that the mean first exit time was always lower for Fermionicvolume excluded diffusion and one might interpret this as volume exclusion biasing the net movement of the rightmost particle towards the right. However, this intuition is incomplete. In particular with biased diffusive transport, we observed the mean first exit time was always higher for Fermionic-volume excluded diffusion. One might attempt to intuitively understand this by considering the prospect that in Bosonic diffusion the bias, which was towards the exit site, allows more particles to be found in the rightmost boxes of the chain, increasing the likelihood that one of them exits, whereas accumulations of particles at the same site close to the exit is not possible for volume-excluded diffusion.

The conflicting predictions highlight that such intuition is too simple. Instead, both mechanisms underlying the intuitive reasoning are most likely relevant and thus whether volume exclusion enhances or inhibits the exit of particles in single line diffusion relies on a relative balance between at least two different aspects of the stochastic dynamics. Hence even predicting trends requires quantitative modeling rather than qualitative reasoning, emphasising that the small single file diffusion systems systems we have investigated are complex, especially due to exclusion. Nonetheless, this complexity is accommodated systematically, intuitively and with generality via matrix representations of ladder operators with anti-commutation relations and quasi-Hamiltonians in the Fock space formalism.

\section{Appendix A: Master equation with volume exclusion}

Here we consider the simplest example how volume exclusion is included in the chemical master equation (1), with three boxes and two particles. Then we have only three possible configurations: $\eta_{1}=\{1,1,0\}, \eta_{2}=$ $\{1,0,1\}$, and $\eta_{3}=\{0,1,1\}$. Let us consider the time evolution of the first configuration $\eta_{1}$ from Eqn. (1)

$$
\frac{\partial \mathcal{P}\left(\eta_{1}, t\right)}{\partial t}=T_{\eta_{2} \rightarrow \eta_{1}} \mathcal{P}\left(\eta_{2}, t\right)-T_{\eta_{1} \rightarrow \eta_{2}} \mathcal{P}\left(\eta_{1}, t\right)
$$

where Eqn. (2) gives us that

$$
T_{\eta_{2} \rightarrow \eta_{1}}=D_{3 \rightarrow 2}
$$

and Eqn. (3) yields

$$
\begin{aligned}
T_{\eta_{1} \rightarrow \eta_{2}} & =\left[(1-\gamma) D_{1 \rightarrow 2}+(1-\gamma) D_{2 \rightarrow 1}\right. \\
& \left.+D_{2 \rightarrow 3}\right] .
\end{aligned}
$$

The departures from $\eta_{1}$ related to the transition rates $D_{1 \rightarrow 2}$, and $D_{2 \rightarrow 1}$ are not permissible due to volume exclusion. Nevertheless, by taking into account the last term in Eqn. (3), with $\gamma=1$ as is the case with volume exclusion, these terms are suppressed, so that $T_{\eta_{1} \rightarrow \eta_{2}}=D_{2 \rightarrow 3}$, yielding the master equation

$$
\frac{\partial \mathcal{P}\left(\eta_{1}, t\right)}{\partial t}=D_{3 \rightarrow 2} \mathcal{P}\left(\eta_{2}, t\right)-D_{2 \rightarrow 3} \mathcal{P}\left(\eta_{1}, t\right)
$$

\section{ACKNOWLEDGMENTS}

This work was partially supported by $\mathrm{CNPq}$ and FACEPE (Brazilian agencies) .
[1] A. W. F. Edwards, Pascal's Problem - The gambler's ruin, International Statistical Review, 51, 73-79 (1983).

[2] P. J. Flory, Spatial Configuration Of Macromolecular Chains, Nobel lecture (1974).

[3] A. Tremel, A. Cai, N. Tirtaatmadja, B. D. Hughes, G. W. Stevens, K. A. Landman, A. J. O'Connor, Cell migration and proliferation during monolayer formation and wound healing, Chemical Engineering Science, 64, 247253 (2009).

[4] A. Bancaud, Molecular crowding affects diffusion and binding of nuclear proteins in heterochromatin and reveals the fractal organization of chromatin, EMBO J., 28, 3785-3798 (2009).

[5] Q. H. Wei, C. Bechinger, P. Leiderer, Single-file diffusion of colloids in one-dimensional channels, Science, 287, 625-627 (2000).

[6] S. Berneche, B. Roux, Energetics of ion conduction through the K+ channel, Nature, 414, 73-77 (2001).

[7] I. Goychuk, P. Hanggi, Ion channel gating: A firstpassage time analysis of the Kramers type, PNAS, 99, 
3552-3556 (2002).

[8] Paul C. Bressloff, Jay M. Newby, Stochastic models of intracellular transport, Rev. Mod. Phys., 85, 135-196 (2013).

[9] Matthijs R. Panman, Pavol Bodis, Daniel J. Shaw, Bert H. Bakker, Arthur C. Newton, Euan R. Kay, Albert M. Brouwer, Wybren Jan Buma, David A. Leigh, Sander Woutersen, Operation Mechanism of a Molecular Machine Revealed Using Time-Resolved Vibrational Spectroscopy, Science, 328, 1255-1258 (2010).

[10] Alessandro Taloni, Michael A. Lomholt, Langevin formulation for single-file diffusion, Phys. Rev. E, 78, 051116 (2008).

[11] M. Bruna, S. J. Chapman, Diffusion of Finite-Size Particles in Confined Geometries, Bulletin of Mathematical Biology, 76, 947-982 (2014).

[12] M. Bruna, S. J. Chapman, Diffusion of multiple species with excluded-volume effects, J. Chem. Phys., 137, 204116 (2012).

[13] Christian A. Yates, Andrew Parker, Ruth E. Baker, Incorporating pushing in exclusion-process models of cell migration, Phys. Rev. E, 91, 069902 (2015).

[14] L. Dyson, R. E. Baker, The importance of volume exclusion in modelling cellular migration, J. Math. Biol., 71, 691-711 (2015).

[15] C. Rodenbeck, J. Karger, K. Hahn, Calculating exact propagators in single-file systems via the reflection principle, Phys. Rev. E, 57, 4382-4397 (1998).

[16] L. Lizana, T. Ambjoernsson, Single-file diffusion in a box, Phys. Rev. Lett., 100, 200601 (2008).

[17] P. M. Centres, S. Bustingorry, Effective EdwardsWilkinson equation for single-file diffusion, Phys. Rev. E, 81, 061101 (2010).

[18] P. R. Taylor, R. E. Baker, M. J. Simpson, C. A. Yates, Coupling volume-excluding compartment-based models of diffusion at different scales: Voronoi and pseudocompartment approaches, Journal of The Royal Society Interface, 13, 2016336 (2016).

[19] P. R. Taylor, C. A. Yates, M. J. Simpson, R. E. Baker, Reconciling transport models across scales: The role of volume exclusion, Phys. Rev. E, 92, 040701 (2015).

[20] E. Barkai, R. Silbey, Theory of Single File Diffusion in a Force Field, Phys. Rev. Lett., 102, 050602 (2009).

[21] A. Ryabov, Single-file diffusion in an interval: First passage properties, J. Chem. Phys., 138, 154104, (2013).

[22] M. Doi, Second quantization representation for classical many-particle system, J. Phys. A: Math. Gen., 9, 1465-77 (1976); see also: M. Doi, Stochastic theory of diffusioncontrolled reaction, J. Phys. A: Math. Gen.,9, 1479-95 (1976).

[23] P. Grassberger, M. Scheunert, Fock-Space Methods for Identical Classical Objects Fortschritte der Physik, 28, $547-5$

[24] L. Peliti, Path integral approach to birth-death processes in a lattice, J. Phys. 46, 1469 (1985).

[25] F. C. Alcaraz, M. Droz,M. Henkel, and V. Rittenberg, Reaction-diffusion processes, critical dynamics, and quantum chains, Ann. Phys. (NY) 230, 250 (1994).

[26] D. C. Mattis and M. Lawrence Glasser, The uses of quantum field theory in diffusion-limited reactions, Rev. Mod. Phys. 70, 979 (1998).

[27] U. C. Tauber, M. Howard, and B. P. Vollmayr-Lee, Applications of field-theoretic renormalization group methods to reaction-diffusion problems, J. Phys. A 38, R79
(2005).

[28] M. Sasai and P. G. Wolynes, Stochastic gene expression as a many-body problem, Proc. Natl. Acad. Sci. USA 111, 2374 (2003).

[29] P. J. Dodd and N. M. Ferguson, A many-body field theory approach to stochastic models in population biology, PLoSONE 4, e6855 (2009).

[30] Fernando A. N. Santos, Hermes Gadelha, Eamonn A. Gaffney, Fock space, symbolic algebra, and analytical solutions for small stochastic systems, Phys. Rev. E, 92, 062714 (2015).

[31] H. A. Araújo, M. O. Lukin, M. G. E. da Luz, G. M. Viswanathan, F. A. N. Santos, E. P. Raposo, Revisiting Lévy flights on bounded domains: a Fock space approach, J. Stat. Mech., 083202 (2020).

[32] J. C. Baez and J. D. Biamonte, Quantum Techniques in Stochastic Mechanics (World Scientific 2018); also available in arXiv:1209.3632.

[33] A. A. Lushnikov, Binary Reaction $1+1 \rightarrow 0$ in one dimension, Zh. Eksp. Teor. Fiz., 91, 1376-1386 (1986).

[34] C. Moler, C. Van Loan, Nineteen Dubious Ways to Compute the Exponential of a Matrix, Twenty-Five Years Later, SIAM Review, 45, 3 (2003).

[35] N. G. van Kampen, Stochastic Processes in Physics and Chemistry, North Holland, 2007.

[36] G. M. Schütz, Exact Solution of the Master Equation for the Asymmetric Exclusion Process. Journal of Statistical Physics, 88, 427445 (1997).

[37] L. Tonks, The complete equation of state of one, two and three-dimensional gases of hard elastic spheres, Phys . Rev., 50 955-963 (1936).

[38] A. Taloni, O. Flomenbom, R. Castaneda-Priego, F. Marchesoni, Single file dynamics in soft materials, Soft Matter, 13, 1096-1106 (2017).

[39] C. S. O. Yokoi, A. Hernandéz-Machado, L. RamírezPiscina, SOME EXACT RESULTS FOR THE LATTICE COVERING TIME PROBLEM, Phys. Lett. A, 145, 82-86 (1996).

[40] T. Hillen, K. J. Painter, A user's guide to PDE models for chemotaxis, J. Math. Biol., 58, 183-217 (2009).

[41] Louise Dyson, Philip K. Maini, Ruth E. Baker, Macroscopic limits of individual-based models for motile cell populations with volume exclusion, Phys. Rev. E, 86, 031903 (2012). 